\title{
A New Crescent-shaped Spindle for Drug Dissolution Testing-But Why a New Spindle?
}

\author{
Saeed A. Qureshi, Ph.D. \\ Senior Research Scientist \\ Banting Research Centre (A/L 2202C1) \\ Therapeutic Products Directorate \\ Health Products and Food Branch, Health Canada \\ Ottawa, Canada K1A OL2.
}

A ssessment of drug dissolution from solid oral dosage forms such as tablets and capsules is an established practice, and an integral part of pharmaceutical product development and quality evaluation. The rationale for conducting such a test is based on the fact that for a drug to be absorbed from gastrointestinal (Gl) tract to systemic circulation, it must be released from the product and dissolved in aqueous based GI tract fluid. In general, without dissolution in aqueous based medium, absorption of the drug in the body may not occur, resulting in lack of anticipated therapeutic effects. Thus drug and drug product dissolution characteristics may directly be related to efficacy of a pharmaceutical product.

Considering its critical importance and extensive use, the testing aspect for measuring dissolution is surprisingly simple in concept and practice. In fact, drug dissolution measurement may be considered as a specific form of solubility measurement. However a critical difference between solubility determination and dissolution testing is that solubility is measured once the solution becomes saturated, a single point answer, but dissolution is measured at single and/or multiple times and usually below saturation. Commonly, solubilities are determined in pure solvents (aqueous solution or organic) at room temperature $\left(20^{\circ} \mathrm{C}\right)$ in a beaker or Erlenmeyer flask with a magnetic stirrer. Drug dissolution is measured at $37^{\circ} \mathrm{C}$ in water or aqueous based buffers ( $\mathrm{pH}$ range of 1 to 7 ) in round bottom containers with special stirrers known as Paddle and Basket [1]. A schematic representation of dissolution vessels and stirrers is shown in Figure 1. Generally known as dissolution apparatuses, they are commercially available with accessories to conduct testing under precisely controlled mechanical and operational parameters.

Generally, drug dissolution tests are conducted using Paddle or Basket apparatuses containing 900 $\mathrm{mL}$ of medium, with spindle rotation speeds between 50 and 150 rpm, most often 50 or 100 $\mathrm{rpm}$. The choice of spindle type and rpm appears to be based on traditional use rather than from scientific rationale. The tests are conducted for various durations from 15 minutes to 24 hours, with frequent sampling, depending on the nature of products. The results are reported as cumulated percent drug dissolved in appropriate times, e.g., $75 \% \mathrm{Q}$ in 45 minutes.

Based on experimental details and observations during the product development phase, the test, or a simpler version of the test, becomes a quality control tool to ascertain lot-to-lot consistency of drug release. Up to this stage there are generally no issues in explaining the need for dissolution testing, conducting it and reporting the results. This is where simplicity of the drug dissolution testing ends.

The main difficulty and complexity in dissolution testing is not in conducting the experiments, but obtaining reproducible results and interpreting and relating the results to product attributes and their biological response (e.g. bioavailability of drug), commonly referred to as in vitro/in vivo correlation (IVIVC). This is where no, or limited, successes have been achieved.

Unfortunately, this issue has been with us since the early days, with the hope that if enough controls and strict guidance for conducting dissolution studies are established, these shortcomings will either be eliminated or at least be controlled.

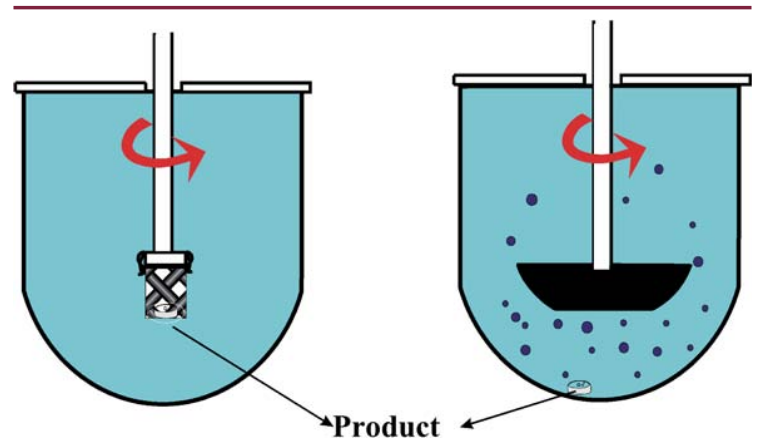

Figure 1. Schematic representations of Basket (USP 1) and Paddle (USP 2) Apparatus 
Therefore, there are an extended number of controls suggested for mechanical specifications [2], deaeration of dissolution media [3] and others [4-5]. There are an impressive number of guidances from US FDA concerning how to conduct relevant dissolution studies [6-10]. However, apparently, we are still struggling with the issues such as whether the dissolution technique is a reproducible technique [11-16] that can produce dissolution data which are relevant to pharmaceutical attributes [17-18] and in turn to biological endpoints [19-20].

The lack of this resolution appears to be due to the fact that there have never been systematic attempts made to evaluate the robustness of the technique and its operational relevance to the physiological environment for which it is utilized. This may be the cause of the lack of success of the apparently sound concept of dissolution testing.

In our laboratory, we became aware of the issue when as a regulatory organization, we tried to set reproducibility standards for dissolution testing between 5 to $10 \%$ CV (coefficient of variation) based on USP Calibrator Tablets testing. Unfortunately by analyzing the archival dissolution results of Calibrator Tablets, obtained from Canadian pharmaceutical manufacturers, it became apparent that drug dissolution testing is a highly variable technique. Therefore, setting standards with 5 to $10 \%$ CV may not be possible [16]. Conclusions drawn from the report suffered a drawback that data received and analyzed was archival and tests were conducted at different times and in somewhat uncontrolled or non-standardized experimental conditions such as choice of deaeration methods. Therefore, it was decided to conduct a study following a common and standard protocol. As a participant of multilaboratory studies for the assessment of quality of drug products, a study was conducted under the direction of Laboratory and Medicinal Control (LMC) Section of International Pharmaceutical Federation (FIP) with the participation of 33 laboratories from 30 countries. The study confirmed the earlier published observations that dissolution testing is indeed a highly variable technique and in some cases one can expect variability of $37 \%$ CV [18]. Later, USP also conducted evaluation of dissolution results of Calibrator Tablets similar to our earlier study (with archival data) and confirmed our findings that one can find a CV of $38 \%$ from a perfectly acceptable apparatus [21].

Before seeking a solution to address the issue of high variability of results, we focused our attention towards determining what might be the potential cause of the problem. There were reports in the literature indicating that irregularities in round-bottom shaped vessels (although still remaining within specified tolerances), positioning of tablets and accumulation of the disintegrating material at the bottom of the vessel, all may cause variation in results [22-23].

Considering these observations as potential problem sources, we focused our attention on the aspect of the hydrodynamics within the dissolution vessel. It was speculated that if one would assume laminar flow in the dissolution vessel then that would result in highly variable flow in the curved portion of the vessel. The particular significance of this effect will be at the bottom of the vessel. Thus variable interaction between product and medium should produce highly variable results. To simulate this effect of variable hydrodynamics, a metal strip based procedure was proposed. The idea behind the metal strip concept was that, not only will it simulate variation in vessel dimensions, within accepted tolerances, but it will also push the positioning of the tablets off-center and enhance spread of the disintegrated material. This will force product into a more stirred area, resulting in significantly higher results than in vessels without the metal-strip. Indeed the results were very different with, or without, the metal strip; thus confirming our hypothesis that variable hydrodynamics exist in the dissolution vessel and can result in highly variable results [24].

Recently this hypothesis of variable hydrodynamics and its potential effect on product dissolution appears to be confirmed based on a computer simulated model of hydrodynamics in the dissolution vessels [14-15]. Therefore, it appears that by the nature of variable hydrodynamics in the vessel, one has to expect highly variable dissolution results. It is just an artifact of the technique. Furthermore, laminar flow patterns and accumulation of disintegrated material at the bottom of the vessel (cone formation) provide a stirring environment, which would not be in line with the stirring and mixing of the Gl tract. Gl tract physiology provides turbulent flow and forces the product to move and spread. Therefore, it would be safe to conclude that since the hydrodynamics and product-medium interaction are significantly different, results from in vitro dissolution testing should not be expected to correspond to in vivo results. This observation may explain, in part, the general lack of successful in vivo relationships. This view appears to support the observation of Repta [20], with regard to in vitro-in vivo studies that were conducted at the University of Maryland in collaboration with US FDA [e.g. see 25] that in spite of developing numerous products with various dissolution characteristics, no corresponding differences in vivo results could be observed.

Furthermore, it was also speculated that due to accumulation of disintegrated products at the centre, the Paddle spindle may inaccurately characterize the in vitro dissolution characteristic of the product i.e. a fast release product will appear as slow-release. 
Thus, until and unless hydrodynamics in dissolution vessels can be improved and/or become better related to that of the Gl tract, conducting useful and relevant dissolution studies will not be possible. With these observations in mind, it was hypothesized that a modified spindle may help in addressing the artifact of the Paddle spindle by providing an improved mixing and stirring environment in the vessel.

After trying several different variants, a design that appears to fulfill the requirements was found. A schematic and a photographic representation of the proposed spindle are shown in Figure 2. The spindle has a curved shape corresponding to the bottom of the vessel. The metallic coil part does not touch the surface of the vessel. The gap between the metal part and bottom of the vessel is filled with filaments. Unlike the gap between the vessel and the spindle for the USP Paddle Apparatus, the crescent-shaped spindle does not have such a gap. The filament ends touch the surface of the vessel and rake through the material, or move the product if not disintegrated, at the bottom of the vessel. The applicability and usefulness of this spindle in comparison with the USP Paddle has been established by testing a number of products including amoxicillin capsules, ASA bolus and multivendor carbamazepine tablet products. The results obtained reflect significant improvements over those obtained with the Paddle spindle, i.e. relevancy to product attributes and physiological behavior [26].

It is to be noted that most problems described here are related to the round bottom shape of the vessel. Although not tested, similar hydrodynamics problems would be expected with the Basket spindle and could perhaps even be exaggerated due to added variances resulting from the movement of the product within the Basket and variable

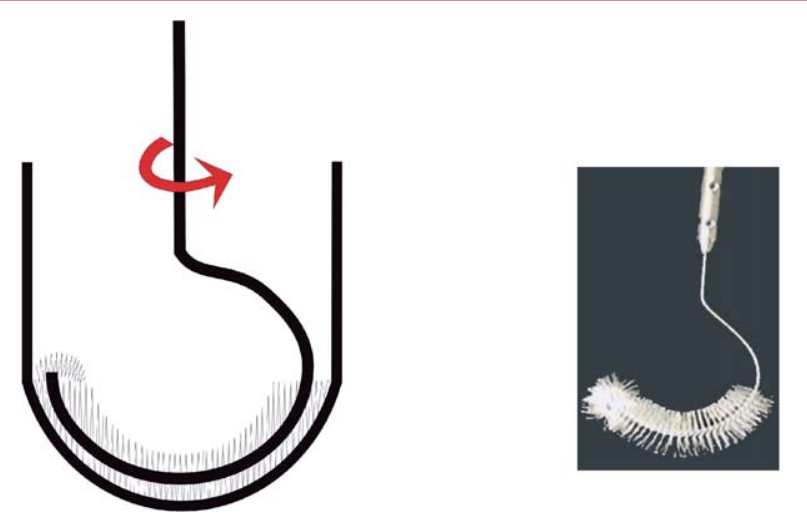

Figure 2. Schematic and pictorial representation of the crescent-shaped spindle. The lower part of the rod (the T-head) as used with the USP Paddle spindle is substituted with the crescent-shaped brush. The crescent-shaped component is attached to the rod with 2 set screws as shown in the photograph. The new spindle may be used with any USP Apparatuses 1 and 2. expression of disintegrated particles from the Basket. In addition, the USP Paddle and Basket Apparatus that provide minimal turbulence within the vessel resulting in stagnation of material, thus may show sensitivity to vibration effect, as commonly speculated. This potential sensitivity to vibration is a weakness of the current apparatus that may lead to high variability and unpredictability (failures) in results. The crescent-shaped apparatus does not allow stagnation of the material, thus is expected to be free from potential vibration effects, providing a more rugged and stable environment for dissolution testing.

Further studies are in progress to support the general applicability of the new spindle in place of the USP Paddle (and possibly the Basket spindle) for improved drug dissolution testing. An interesting and useful application of the crescent-shaped spindle is described here using USP Prednisone Calibrator and diltiazem tablets as test products.

Results from experimental studies are provided to describe how poor and variable hydrodynamics with the Paddle spindle can provide potentially inaccurate dissolution characteristics of products and how the proposed new spindle may provide improved dissolution results to accurately reflect product characteristics.

In these experiments, dissolution characteristics were determined for two different types of conventional(immediate-) release tablet products, i.e. $10 \mathrm{mg}$ Prednisone Calibrator and $60 \mathrm{mg}$ diltiazem. Based on the information described in the literature, the drug release characteristics of these products may be described as follows. USP Prednisone Calibrator Tablets - fast disintegrating tablet resulting in dense particulate material, which settles at the bottom of the vessel during drug dissolution testing. It is a fast drug release product, but using Paddle apparatus, due to the accumulation of disintegrated material at the bottom of the vessel, would appear to show slower drug release [27]. Diltiazem Tablets - a conventional (immediate) release product but expected to have a slow dissolution with two point tolerances at 30 minutes and 3 hours [28]. Further, based on observations during experiments, it was noted that tablets did not disintegrate for most of the testing (up to about $2 \mathrm{hr}$ ). After that time, tablets disintegrated into smaller particles, which settled at the bottom of the vessel.

Mean $(n=6)$ percent drug release profiles using the Paddle and crescent-shaped spindle are compared in Figures 3 (prednisone product) and 4 (diltiazem product). The tests were conducted at 50 and $75 \mathrm{rpm}$ using the Paddle spindle for Prednisone and diltiazem tablets, respectively. However, $25 \mathrm{rpm}$ was used for the crescent-shaped spindles for both products. With both spindles, water, 500 $\mathrm{mL}$ for Prednisone and $900 \mathrm{~mL}$ for diltiazem, was used as the dissolution medium. The quantitation was achieved 


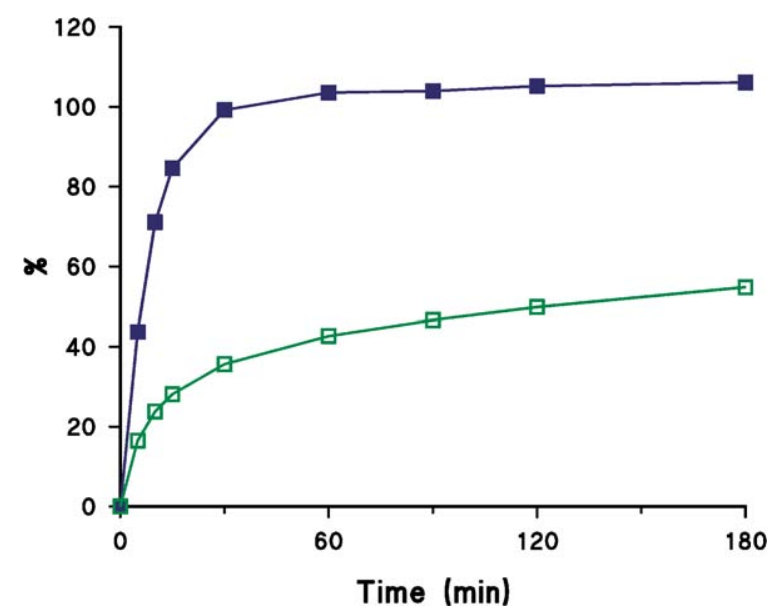

Figure 3. Mean ( $n=6)$ percent drug release profiles of USP $10 \mathrm{mg}$ Prednisone Calibrator Tablets with USP Paddle spindle at $50 \mathrm{rpm}$ ( - $\square$-) and the crescent-shaped spindle at $25 \mathrm{rpm}$ (-口-).

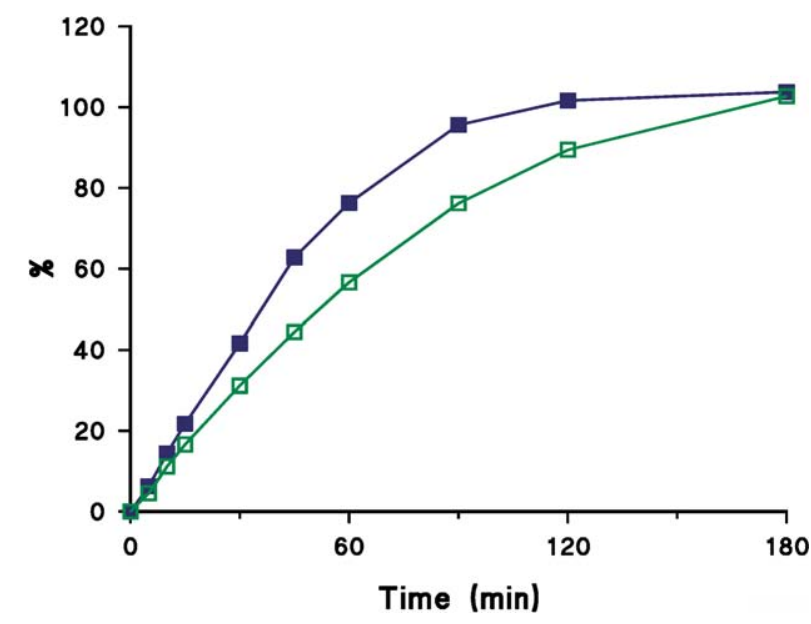

Figure 4. Mean ( $n=6)$ drug release profiles of a $60 \mathrm{mg}$ commercially available conventional-release diltiazem tablet product with the USP Paddle spindle at $75 \mathrm{rpm}$ (- $\square$-) and the crescent-shaped spindled at $25 \mathrm{rpm}$ (-口-).

using an online UV diode array spectrophotometer, set at $242 \mathrm{~nm}$ for Prednisone and $240 \mathrm{~nm}$ for diltiazem.

Drug dissolution characteristics of Prednisone Tablets show significant differences using the two types of spindles. The reason is that when using the Paddle spindle, after disintegration, the disintegrate settles at the bottom centre of the vessel, thus providing limited interaction (dissolution). On the other hand, using the crescentshaped spindle, with better stirring and mixing and avoiding cone formation, provides improved productmedium interaction. This results in higher dissolution and a more appropriate reflection of a fast-dissolving drug product. The dissolution behavior of this product using the spindles is shown in Figure 5. The lack of product-medium
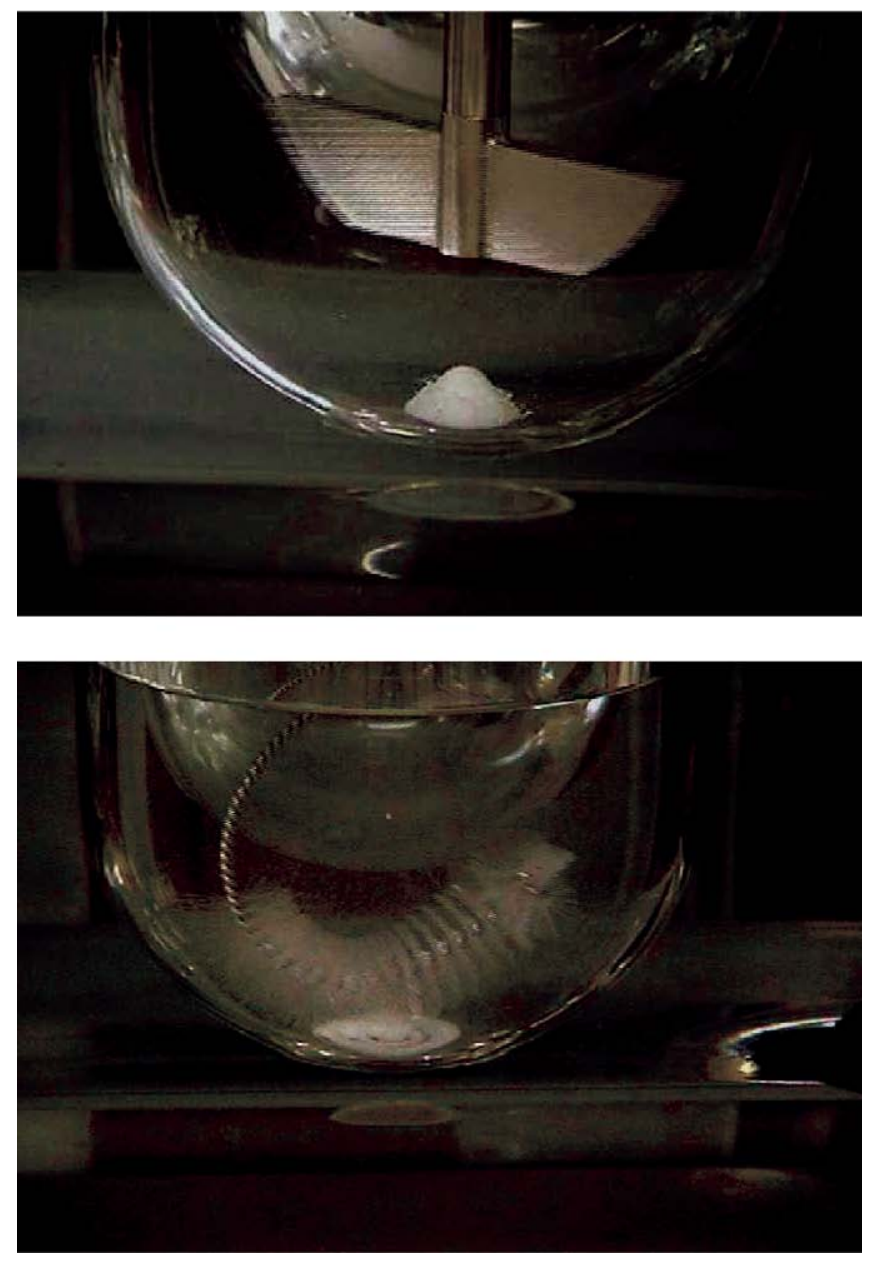

Figure 5. Photographic images of the test product (USP Prednisone Calibrator Tablets) behavior using the USP Paddle spindle (50 rpm) and the crescent-shaped spindle (25 rpm).

interaction using the Paddle spindle and the improved interaction with the crescent-shaped spindle are evident from the pictures. Therefore, the drug release characteristics were spindle dependent. Obviously, the Paddle spindle would not be the choice as an appropriate spindle for this or similar products, as it appears to inaccurately characterize the fast-release product as a slow release.

On the other hand, drug dissolution differences for diltiazem tablets, using the two spindles, were relatively less dramatic. In this case, the tablets did not disintegrate, thus in both cases surface area remained more or less the same. The differences in dissolution results appears to be because, with the Paddle spindle, tablets remain stationary at various positions in the vessels, while with the crescentshaped spindle they moved with the spindle. In the case of the Paddle, the tablet surface touching the vessel offers no or limited interaction with medium [29] compared with 
the crescent-shaped spindle where tablets were in motion and provide dissolution from all surfaces resulting in higher results. Therefore, testing with the Paddle spindle resulted in slower dissolution than is the true release capability of the product. Thus, for at least two different reasons, dissolution testing using the Paddle spindle is expected to provide slower results for both types of products (disintegrating and non-disintegrating) failing to accurately reflect the product release capabilities.

An even more significant observation from this study is that dissolution of diltiazem tablets vs. Calibrator Tablets with the Paddle, using suggested experimental conditions, results in an inaccurate comparison i.e. slower release diltiazem appears faster than the Prednisone Tablets. However, in reality the drug release characteristics of these products are the opposite. This discrepancy is due to the variable hydrodynamics and poor stirring environment in the vessel using the Paddle spindle. The crescent-shaped spindle, with a better stirring and mixing environment, provides a truer reflection of these product dissolution characteristics.

In conclusion, due to variable hydrodynamics and poor mixing and stirring characteristics, the Paddle spindle may provide variable results and an inaccurate reflection of product release characteristics (some fast dissolving products will appear to be slow release). Further, testing with the Paddle spindle is unlikely to provide bio-relevant results, due to the different mixing environment of the Gl tract. These artifacts, resulting from the Paddle-vessel interaction, may be alleviated by modifying the spindle to provide improved hydrodynamics, resulting in improved product characterization. The crescent-shaped spindle appears to fulfill this need by addressing the artifacts of the Paddle and providing improved dissolution testing and product characterization.

\section{References}

1. USP. " General Chapter <711> Dissolution,"USP 27 The United States Pharmacopeial Convention, Inc. Rockville, MD., pp. 2303-4 (2004).

2. PHRMA. Dissolution Calibration: Recommendations for Reduced Chemical Testing and Enhanced Mechanical Calibration. Pharmacopeial Forum, 26, 1149-66 (2000).

3. USP. General Chapters $<1092>$ The Dissolution Procedure: Development and Validation. Pharmacopeial Forum. 30, 351-363 (2004).

4. Mirza, T., Grady, L.T. and Foster, T.S. Merits of Dissolution System Suitability Testing: Response to PhRMA's Proposal on Mechanical Calibration, Pharmacopeial Forum, 25(4), 1167-9 (2000).

5. Gray, V.A., Hubert, B.B. and Krasowski, J.A. Calibration of Dissolution Apparatuses 1 and 2 - What to Do
When Your Equipment Fails. Pharmacopeial Forum, 20, 8571-2 (1994).

6. Dissolution Testing of Immediate Release Solid Oral Dosage Forms, http://www.fda.gov/cder/ guidance/1713bp1.pdf

7. Extended Release Oral Dosage Forms: Development, Evaluation, and Application of In Vitro/In Vivo Correlations, http://www.fda.gov/cder/ guidance/1306fnl.pdf

8. Waiver of In Vivo Bioavailability and Bioequivalence Studies for Immediate Release Solid Oral Forms Containing Certain Active Moieties/Active Ingredients Based on Biopharmaceutics Classification System, http://www.fda.gov/cder/guidance/ 3618fnl.pdf

9. SUPAC-IR: Immediate-Release Solid Oral Dosage Forms: Scale-Up and Post-Approval Changes: Chemistry, Manufacturing and Controls, In Vitro Dissolution Testing, and In Vivo Bioequivalence Documentation, http://www.fda.gov/cder/ guidance/cmc5.pdf

10. SUPAC-MR: Modified-Release Solid Oral Dosage Forms: Scale-Up and Post-Approval Changes: Chemistry, Manufacturing and Controls, In Vitro Dissolution Testing, and In Vivo Bioequivalence Documentation, http://www.fda.gov/cder/guidance/1214fnl.pdf

11. Cox, D.C., Wells, C.E., Furman W.B., Savage, T.S. and King, A.C., Systematic Error Associated with Apparatus 2 of the USP Dissolution Test II: Effects of Deviations in Vessel Curvature From That of a Sphere, J. Pharm. Sciences. 71: 395- 399, (1982).

12. McCormick, T.J., Industry Perspective on Dissolution Apparatus Calibration. Dissolution Technology. 2: 12-15 (1995).

13. Moore, T.W. and Cox, D.C. An Open Response to the USP Subcommittee on Dissolution and Bioavailability (DBA). Pharmcopeial Forum. 23, 4581-2 (1997).

14. Kukura, J., Baxter, J.L., Muzzio F.J. Shear Distribution and Variability in the USP Apparatus 2 under Turbulent Conditions. Int. J. Pharm. 26: 9-17 (2004).

15. McCarthy, L. G., Kosiol, C., Healy, A.M., Bradley, G., Sexton, J.C., Corrigan, O.I. Simulating the Hydrodynamic Conditions in the United States Pharmacopeia Paddle Dissolution Apparatus. AAPS PharmSciTech; 4, 172-187 (2003).

16. Qureshi, S.A. and McGilveray, I.J. A Critical Assessment of the USP Dissolution Apparatus Suitability Test Criteria. Drug Develop. Ind. Pharm. 21, 905-92 (1995).

17. Qureshi, S.A. and McGilveray, I.J. Assessment of Pharmaceutical Quality of Furosemide Tablets From Multinational Markets. Drug Develop. Ind. Pharm., 24, 995-1005 (1998). 
18. Qureshi, S.A. and McGilveray, S.A. Typical variability in drug dissolution testing: study with USP and FDA calibrator tablets and a marketed drug (glibenclamide) product. Eur. J. Pharm. Sci., 7: 249-258 (1999).

19. Meyer M.C., Straughn A.B., Mhatre R.M., Shah V.P., Williams R.L., and Lesko L.J. Lack of in vivo/in vitro correlations for $50 \mathrm{mg}$ and $250 \mathrm{mg}$ primidone tablets. Pharm Res. 15, 1085-9 (1998).

20. Repta, A.J, Dissolution specifications for drug products. AAPS Pharmsci. (Suppl.), 2531 (1999).

21. Achanta, A.S., Gray, V.A., Cecil, T.L. and Grady, L.T. Evaluation of the Performance of Prednisone and Salicylic Acid Calibrators. Drug Develop. Ind. Pharm. 21, 1171-1182 (1995).

22. Beckett, A.N., Quach. T.T., Kurs, G.S. Improved hydrodynamics for USP apparatus 2. Dissolution Technol. 3. 1-10 (1996).

23. Cox, D.C., Wells, C.E., Furman W.B., Savage, T.S. and King, A.C. Systematic Error Associated with Apparatus 2 of the USP Dissolution Test II: Effects of Deviations in Vessel Curvature From That of a Sphere, J. Pharm. Sciences. 71, 395-399 (1982).

24. Qureshi, S.A. and Shabnam, J., Cause of high variability in drug dissolution testing and its impact on setting tolerance. Eur. J. Pharm. Sci., 12, 271-276 (2001).

25. Eddington, N.D., Ashraf, M., Augsburger, L.L., Leslie, J.L., Fossler, M.J., Lesko, L.J., Shah, V.P., Rekhi, G.S. Identification of formulation and manufacturing variables that influence in vitro dissolution and in vivo bioavailability of propranolol hydrochloride tablets. Pharm Dev Technol. 3, 535-47 (1998).

26. Qureshi, S.A. and Shabnam, J. Application of a new device (spindle) for improved characterization of drug release (dissolution) of pharmaceutical products. Eur. J. Pharm. Sci., 19: 291-7 (2003).

27. Moore, T.W. and Cox, D.C. Dissolution testing: collaborative study of the in-house NCDA \#2 dissolution calibrator tablets with USP apparatus 2. Pharmacopeial Forum. 23, 4250-5, 1997.

28. USP. “Diltiazem Hydrochloride Tablets - Monograph", USP 27 - The United States Pharmacopeial Convention, Inc. Rockville, MD., p. 628, 2004.

29. Morihara, M., Aoyagi, N. Kaniwa, N., Katori, N., and Kojim, S. Hydrodynamic Flows Around Tablets in Different Pharmacopeial Dissolution Tests. Drug Develop. Ind. Pharm., 28, 655-662 (2002).

\section{Acknowledgements}

The skillful technical help provided by Dr. M. Vivas is greatly appreciated. Dr. lain McGilveray's help in reviewing the manuscript and for providing valuable suggestions is also greatly appreciated. 